\title{
MODELLING, SIMULATION AND BALANCE CONTROL OF A TWO- WHEELED ROBOTIC MACHINE WITH STATIC VARIATION IN LOAD POSITION
}

\author{
Khaled M K Goher and M O Tokhi \\ Department of Automatic Control and Systems Engineering \\ The University of Sheffield \\ Mappin Street, Sheffield, S1 3JD, \\ United Kingdom. \\ E-mail: k.mourad@shef.ac.uk
}

\section{KEYWORDS}

Wheeled robot, inverted pendulum, balance mode, PD control. Mathematical Modelling, Simulation.

\begin{abstract}
This paper focuses on the design and implementation of a control algorithm for balancing of a two-wheeled robotic machine (TWRM). The machine comprises a rod on an axle incorporating two wheels. The balancing of the rod in the upright position is achieved using a proportional-derivative (PD) controller. The NewtonEuler dynamic formulation is used to develop a mathematical model of the system. Matlab Simulink is used as a simulation environment. The intermediate body (IB) is considered to be in the upright position. An external disturbance force is applied at different locations of the IB in order to test the robustness of the developed controller. A payload is considered, and this is attached at different locations along the rod. Investigations are carried out on the effect of changing the duration and location of disturbance force, and changing the location of the payload on the system in the balancing mode. Two PD controllers are developed, one for the angular position of the rod and one for the cart linear displacement. Simulation results are presented assessing the performances of the controllers.
\end{abstract}

\section{INTRODUCTION}

An inverted pendulum system is an under-actuated mechanical system and inherently open loop unstable with highly non-linear dynamics. It is thus a perfect testbed for the design of a wide range of classical and contemporary control techniques. It has wide ranging applications from robotics to space rocket guidance systems. The concept of balancing a robot is based on the inverted pendulum model. This model has been widely used by researches worldwide in the design and control of wheeled legged robots, etc. (Formal and Martynenko 2005). The inverted pendulum problem is common in the field of control engineering. The uniqueness and wide application of technology derived from this unstable system has drawn interest of many researches and robotics enthusiasts around the world. In recent years, researchers have applied the idea of a mobile inverted pendulum model to various problems, such as designing walking gaits for humanoid robots, robotic wheelchairs and personal transport systems (Kim, et al. 2005). The type of intelligent robot proposed here is a mobile robot with a two wheeled inverted pendulum. This design was chosen because its mechanism has an inherently clumsy motion for stabilizing the robot's body posture. The robot has a body with two wheels for moving in a plane and a head similar to a human head for controlling the motion. Two independent driving wheels are used for position control and for fast motion in a plane without casters.

\section{TWO-WHEELED INVERTED PENDULUM ROBOT}

Several kinds of wheels can be attached to the wheeled mobile robot, but they fall into one of two categories: driving wheels and auxiliary wheels. The driving wheels are rotated to permit the robot to move when torque is applied to the axles. The auxiliary wheels merely ease the movement of the robot and enable its body to be suspended when no torque is applied to the axles. In most wheeled mobile robots at least one wheel is an auxiliary wheel. For a two-wheeled mobile robot, one can ask what would happen if the auxiliary wheels were removed altogether instead of being replaced with something different or instead of improving the performance of the wheels. By removing the auxiliary wheels, the number of wheels attached on the robot would be reduced. Moreover, the mechanical characteristics of the robot would be completely altered because no elements could suspend and balance the robot's body except for the driving wheels. That is, the robot would have to move and balance its body with only two driving wheels (Kim, et al. 2005). The mechanical structure of a robot with only two driving wheels is similar to an inverted pendulum (D'Andrea and Earl 2005).

\section{MATHEMATICAL MODELLING}

The dynamic characterisation of the robot is described in this section by introducing the governing main equations describing the mathematical model of the two-wheeled robotic machine (TWRM). The model is derived based on the Newton-Euler equations of motion. The dynamic model of the whole system consists of two 
separate sub-models, namely: the non-linear model of the inverted pendulum, and a linear model of the permanent magnet (PM) direct current (DC) motor activating the cart.

\section{Linear model of the DC motor}

The robot is powered by two DC motors, Figure 1, where $V_{a}$ represents the applied voltage, $i$ the armature current. The mathematical model of the DC motor is derived and then used in the dynamic model of the robot to provide a relationship between the input voltage to the DC motors and the control signal needed to stabilise the robot.

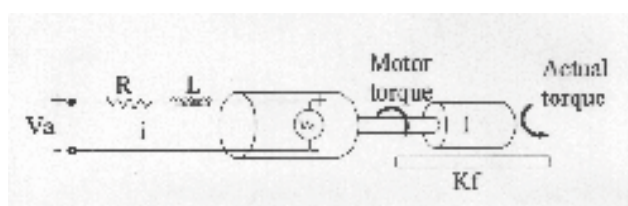

Figure 1 Schematic diagram of a DC motor

Using Figure 1, the following two fundamental equations of motion of the DC motor can be written as:

$$
\frac{d i}{d t}=\frac{R}{L} i+\frac{k_{e}}{L} \omega+\frac{V_{a}}{L}
$$

and

$$
\frac{d \omega}{d t}=\frac{k_{m}}{I_{R}} i-\frac{k_{e}}{I_{R}} \omega-\frac{\tau_{a}}{I_{R}}
$$

where $R$ and $L$ are the armature resistance and inductance, $V_{e}$ represents the winding emf, $I_{R}$ the rotor inertia and $K_{f}$ the friction constant, $\omega$ is the angular velocity of the motor shaft, $k_{e}$ and $k_{m}$ represent the back emf Constant and proportionality constant and $\tau_{a}$ is the motor shaft torque. Equations (1) and (2) are used to build the motor model in the Matlab environment. The inputs to the model are the applied voltage $V_{a}$ and the applied torque $\tau_{a}$. The model outputs are the angular displacement $\theta$ and angular velocity $\omega$ of the motor shaft. Both equations are first order and linear functions of the armature current and velocity of the motor shaft.

\section{Non linear model of the two-wheeled inverted pendulum}

The pendulum and wheel dynamics are analysed separately at the beginning, but this will eventually lead to two equations of motion which completely describe the behaviour of the TWRM.

Consider Figure 2 which represents the free body diagram of the right and left wheels of the robot, Where
$H_{f r}$ and $H_{r}$ are the friction force between the wheel and the ground and the interaction force at the rod and axle interface respectively for the right wheel.

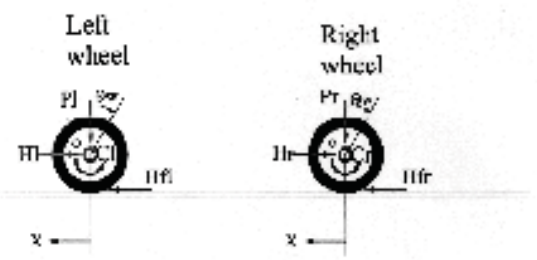

Figure 2 Free body diagrams of robot wheels

The dynamic equation of motion of the wheels can be described as:

$$
\begin{aligned}
& 2\left(M_{w}+\frac{I_{w}}{R_{w}^{2}}\right) \ddot{x} \\
& =-\frac{k_{m} k_{e}}{R R_{w}^{2}} \dot{x}+\frac{k_{m}}{R R_{w}} V_{a}-\left(H_{R}+H_{L}\right)
\end{aligned}
$$

Consider Figure 3 which represents the free body diagram of the IB with the external applied disturbance force, $F$. Applying the Newton's second law of motion in the horizontal direction yields

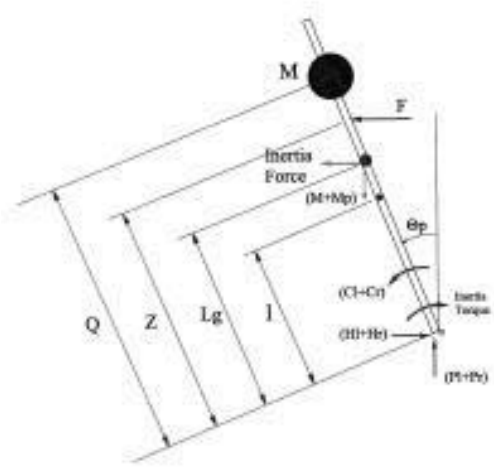

Figure 3 Free body diagrams of the IB

$$
\begin{gathered}
\sum F_{x}=\left(M+M_{p}\right) a_{x} \\
\left(H_{R}+H_{L}\right)-\left(M+M_{p}\right) L_{g} \ddot{\theta}_{p} \cos \theta_{p} \\
+\left(M+M_{p}\right) L_{g} \dot{\theta}_{p}^{2} \sin \theta_{p}-F=\left(M+M_{p}\right) \ddot{x}
\end{gathered}
$$

Considering the sum of the applied forces in a direction perpendicular to the rod gives

$$
\begin{gathered}
\sum F_{\perp p}=\left(M+M_{p}\right) \ddot{x} \cos \theta_{p} \\
\left(H_{R}+H_{L}\right) \cos \theta_{p}+\left(P_{R}+P_{L}\right) \sin \theta_{p} \\
-\left(M+M_{p}\right)\left(g \sin \theta_{p}-L_{g} \ddot{\theta}_{p}\right)-F \cos \theta_{p} \\
=\left(M+M_{p}\right) \ddot{x} \cos \theta_{p}
\end{gathered}
$$


Equating the sum of the applied moments around the global centre of mass yields

$$
\begin{gathered}
\sum M_{o}=I_{g} \alpha \\
\left(-\left(H_{R}+H_{L}\right) \cos \theta_{p}-\left(P_{R}+P_{L}\right) \sin \theta_{p}\right) L_{g} \\
-\left(C_{R}+C_{L}\right)-F \cos \theta_{p}\left(Z-L_{g}\right)=I_{g} \ddot{\theta}_{p}
\end{gathered}
$$

Manipulating the above equations yields the following two first order non-linear differential equations describing the motion of the system under the effect of an applied payload and impact disturbance force on the rod:

$$
\begin{aligned}
\ddot{\theta}_{p} & =\frac{1}{\left(I_{g}+\left(M+M_{p}\right) L_{g}^{2}\right)}\left(\frac{2 k_{m} k_{e}}{R R_{w}} \dot{x}-\frac{2 k_{m}}{R} V_{a}\right. \\
& \left.-\left(M+M_{p}\right) g L_{g} \sin \theta_{p}-\left(M+M_{p}\right) L_{g} \ddot{x} \cos \theta_{p}-F Z \cos \theta_{p}\right)
\end{aligned}
$$

and

$$
\begin{gathered}
\ddot{x}=\frac{1}{\left(2 M_{w}+2 \frac{I_{w}}{R_{w}^{2}}+\left(M+M_{p}\right)\right)}\left(-\frac{k_{m} k_{e}}{R R_{w}^{2}} \dot{x}+\frac{k_{m}}{R R_{w}} V_{a}\right. \\
\left.-\left(M+M_{P}\right) L_{g} \ddot{\theta}_{p} \cos \theta_{p}+\left(M+M_{P}\right) L_{g} \dot{\theta}_{p}^{2} \sin \theta_{p}-F\right)
\end{gathered}
$$

\section{Effect of Changing the Payload Location}

Let the payload, $M$ be located at a variable distance $Q$ from the IB origin $O$, as shown in Figure 3, where $Q$ can be expressed as a factor $y$ multiplied by the rod half length $l$ as follows:

$$
Q=y l
$$

where y can take a value from 0 to 2 according to the position of the payload $M$ from the origin $O$ as follows:

For $M$ to be concentrated at the upper end of the $\operatorname{rod} y=2$,

For $M$ to be concentrated at the mid-span of the $\operatorname{rod} y=1$, and

For $M$ to be concentrated at the lower end of the $\operatorname{rod} y=0$

Changing the payload position will affect both the overall moment of inertia, $I_{g}$ and the location of the global centre of mass of the IB, $L_{g}$. The overall moment of inertia of the IB is affected and modified as:

$$
I_{g}=\frac{M_{p}(2 l)^{2}}{12}+M_{p}\left(L_{g}-l\right)^{2}+M\left(Q-L_{g}\right)^{2}
$$

The location of the global centre of mass of the IB will be affected as:

$$
L_{g}=\frac{\left(M_{p} l+Q M\right)}{\left(M_{p}+M\right)}
$$

\section{BALANCE CONTROL USING PD CONTROLLER}

At this stage the inverted pendulum rod is considered initially in the upright position. With a disturbance force applied to the rod, it will move from that upright position with an angle (measured from that position) proportional to the magnitude and direction of the disturbance force. In order to keep the whole system controlled, two PD controllers are developed; one for controlling the angular position of the rod for returning the rod back to the upright position after a change caused by the disturbance force, and one for keeping the cart wheels within a specified linear position from a specific reference position. The parameters of the two PD controllers are tuned manually to achieve the desired target at the balancing mode.

PD control parameters for the linear position of the wheels were tuned as $K_{p 1}=6.3, K_{D 1}=150$

PD control parameters for the angular position of the rod were tuned as $K_{p 2}=5, K_{D 2}=30$

\section{SIMULATION RESULTS}

Simulations were carried out on the system using the numerical parameters describing the system features, listed in Tables 1 and 2. The simulations presented to highlight the way the vehicle will behave with the effect of changing the values of three different variables and the control effort required to accomplish the required task. Those variables are the level of the applied disturbance force, the duration of the disturbance force and the position of the payload attached to the vehicle rod. The target was to estimate the limits of those variables beyond which the control algorithm will not be able to balance the system around the upright position. The disturbance force was applied after 10 seconds at the start of the simulation and then after each 90 second interval. This interview was determined as the safe limit for repeating such impact forces to the rod. Below such limit, it was found that the developed controller would not completely keep the IB at a stable position.

Table 1: DC Motor technical properties

\begin{tabular}{|l|l|l|}
\hline Parameter & Description & Units \\
\hline$R=3$ & Nominal terminal Resistance & Ohms \\
\hline$L=0$ & Rotor Inductance & $\mathrm{H}$ \\
\hline$K_{e}=0.00607:$ & Back EMF Constant & $\mathrm{VS} / \mathrm{rad}$ \\
\hline$V_{e}=0$ & Back EMF Voltage & Vlots \\
\hline$I_{r}=0$ & Rotor Inertia & $\mathrm{kgm}^{2}$ \\
\hline
\end{tabular}


Table 2: Two-wheeled inverted pendulum properties

\begin{tabular}{|l|l|l|}
\hline Parameter & Description & Units \\
\hline$R_{w}=0.051$ & Wheel Radius & $\mathrm{m}$ \\
\hline$M_{w}=2$ & Wheel Mass & $\mathrm{kg}$ \\
\hline$M_{p}=2$ & Pendulum Mass & $\mathrm{kg}$ \\
\hline$M^{\prime}=50$ & Payload & $\mathrm{kg}$ \\
\hline$I_{w}=0.000039$ & Wheel Inertia & $\mathrm{kgm}^{2}$ \\
\hline$I_{p}=0.0041$ & Pendulum inertia & $\mathrm{kgm}^{2}$ \\
\hline$l=0.07$ & Length to the Pendulum COM & $\mathrm{m}$ \\
\hline
\end{tabular}

\section{Effect of Different Disturbance Levels}

The effect of changing the level of the applied disturbance force is considered in this section. Four levels of disturbance amplitudes including zero to test the validity of the developed controller in coping with external disturbances. The simulations are carried out by considering the disturbance force applied at different positions on the IB of the vehicle. Such assumptions may be useful in applications such as extended height of crane arms if subjected to sudden external forces through their stems especially during motion.

Figures 4, 5 and 6 show the system performance with the disturbance force applied at the upper end, mid-span and lower end of the IB respectively.

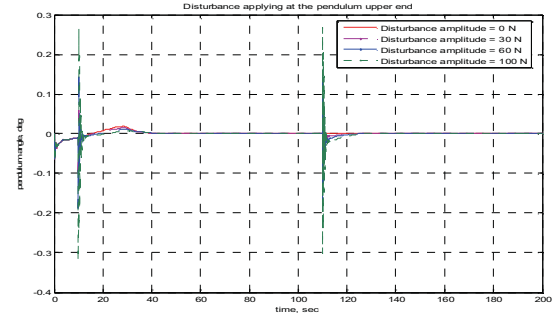

(a) Intermediate body tilt angle

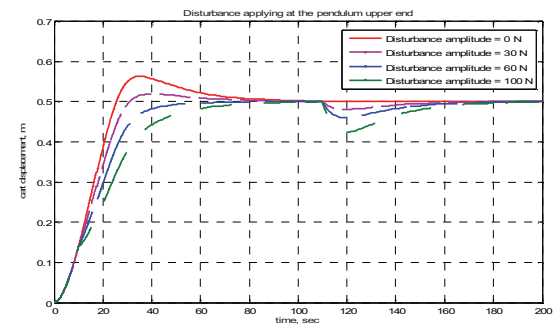

(b) Linear displacement of the vehicle wheels

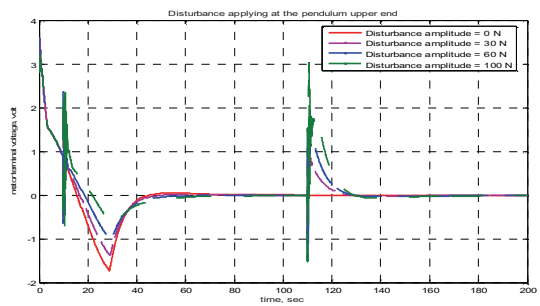

(c) Controller output signal to the system

Figure 4: System performance with disturbance applied at the IB upper end

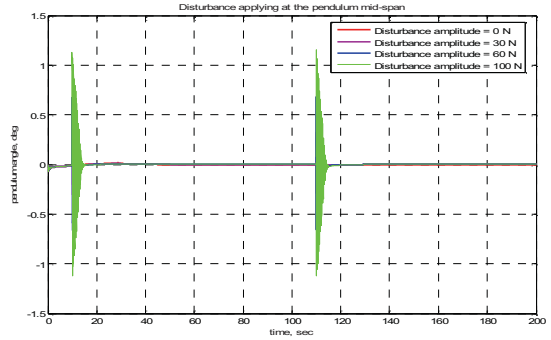

(a) Intermediate body tilt angle

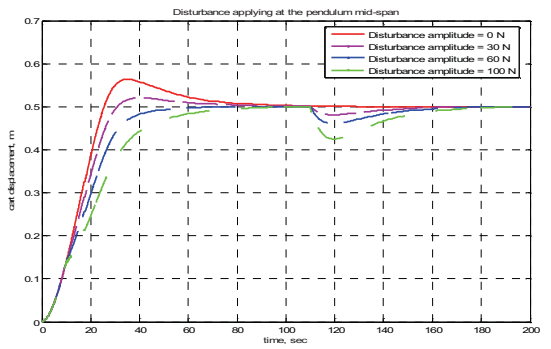

(b) Linear displacement of the vehicle wheels

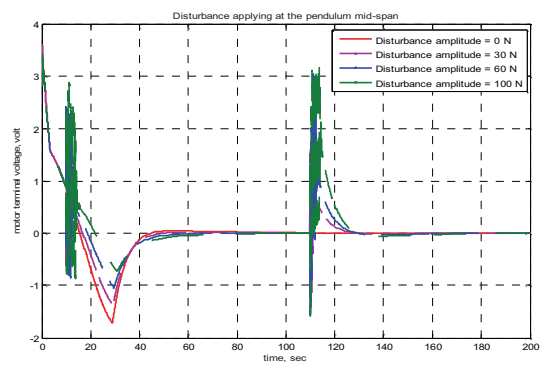

(c) Controller output signal to the system

Figure 5: System performance with disturbance applied at the IB mid-span

It can be noted from Figures 4(a), 5(a) and 6(a) that the higher the level of the disturbance force the longer the fluctuation of the rod angle from the upright position. It can also be noted that the closer the location of the applied disturbance force to the IB origin the harder it is to balance the system as it takes the control system a long time to bring the rod to the target position.

The cart linear displacement is presented in Figures 4(b), 5(b) and 6(b). Increasing the level of the applied disturbance tends to decease the cart overshoot beyond the specified limit. That is because the amount of the applied force acts as a drag force for the cart and slows the cart which in turn increases the rise time of the system response. This phenomenon is clearly repeated when the force is reapplied to the system. The lower the level of the disturbance force the faster the cart in achieving the desired position.

Changing the level of the disturbance force also affected the control effort, as clearly presented through Figures 4(c), 5(c) and 6(c). Higher levels of the applied force tended to increase the time the control signal takes to 
settle down. The location of applying the disturbance force also greatly affected the control behaviour. The closer the position of the force to the rod origin the more the control effort fluctuation and the higher the control effort at the time of applying the force.

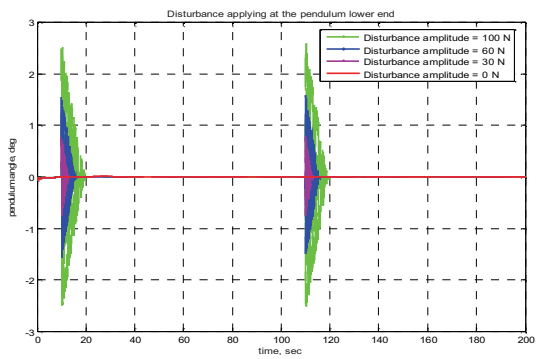

(a) Intermediate body tilt angle

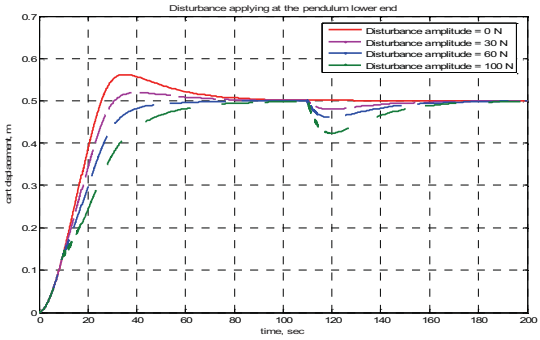

(b) Linear displacement of the vehicle wheels

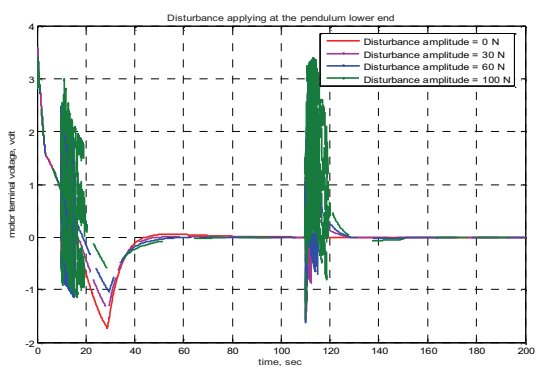

(c) Controller output signal to the system

Figure 6: System performance with disturbance applied at the IB lower end

\section{Effect of Disturbance Duration}

The duration of applying such disturbances is another variable of interest. Four different durations of applying the disturbance force are used to estimate the safe limits beyond which the developed controller will not be able to cope.

Figures 7 and 8 show the IB tilt angle the vehicle displacement, with different duty cycles of a disturbance force of $100 \mathrm{~N}$ and $30 \mathrm{~N}$ respectively. It is noted in Figure 7 that the amount of overshoot in the IB tilt angle increases as the period of the force is increased, and the controller still copes well with such higher periods of time especially for the pendulum tilt angle. The system can recover faster to the desired position with shorter force duty cycles
For the cart linear displacement, as described in Figure 8 , the situation not as good as for the tilt angle; increasing the force duty cycle made the system slower in achieving the target and increased the rise time and decreased the cart overshoot. But for shorter duty cycles, the overshoot tended to be higher which is a characteristic of such impulse disturbance force but accelerated the system behaviour to reach the desired position.

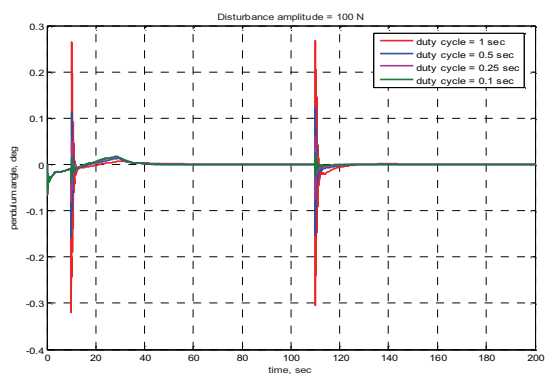

Figure 7 Intermediate body tilt angle

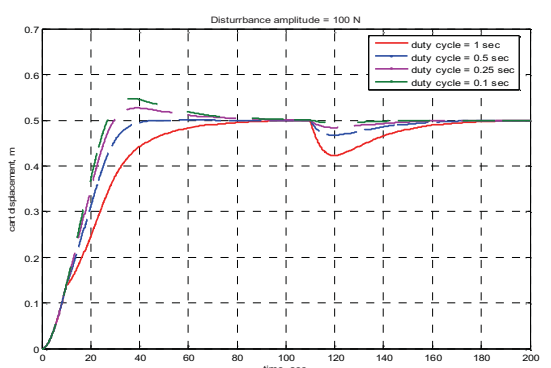

Figure 8 Linear displacement of the vehicle wheels

The control effort is presented in Figure 9. It is noted that the longer the disturbance duty cycle the higher the control effort required and the longer the time for the control signal to settle down.

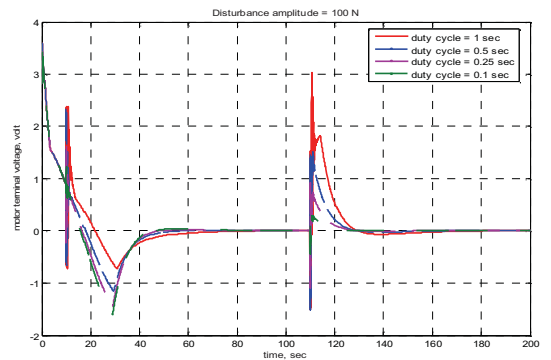

Figure 9 Controller output signal to the system

\section{Effect of Payload Position}

The third variable of interest is the location of the payload attached to the IB. The system behaviour including the IB tilt angle and the cart linear displacement are presented and the control effort required for three different locations of the payload. The load is positioned at upper, mid-span and the lower end of the IB. Changing the location of the load mainly affects the location of the global centre of mass and the 
whole moment of inertia of the pendulum as presented before.

Two different levels of the disturbance force are implemented here with the assumption of the force at the upper end of the rod. Figures 10 and 11 show the system performance with different load positions and disturbance forces of $100 \mathrm{~N}$ and $30 \mathrm{~N}$ respectively.

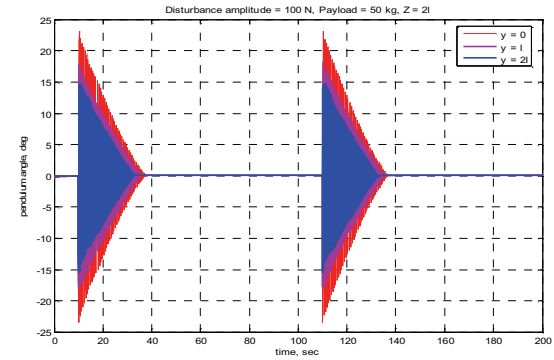

(a) Intermediate body tilt angle

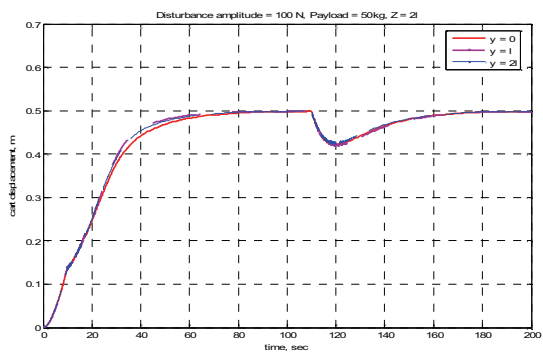

(b) Linear displacement of the vehicle wheels

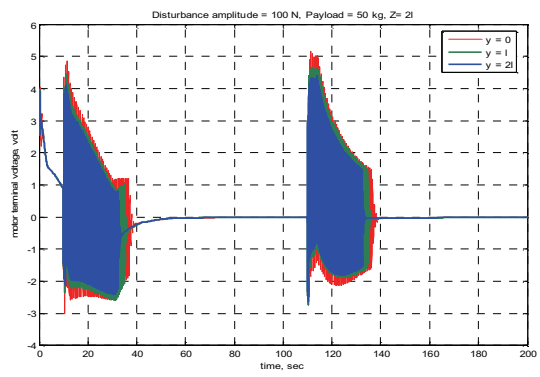

(c) Controller output signal to the system

Figure 10: System performance with load positions and $100 \mathrm{~N}$ disturbance force

It is noted from Figures 10(a) and 11(a) that the closer the payload to the IB origin the harder the balancing in the upright position. More fluctuations of the rod occur as the load is closer to the origin. This phenomenon is similar to the system behaviour for the case of applying the disturbance force at the lower end of the rod as previously described in Figures 6 . Therefore, this means that the harder it is to balance the IB when either the force is applied at lower positions of the rod or the payload is closer to the origin. Hence, the worst and most hard case of balancing is more likely to happen when the payload and disturbance force are both close to the IB origin.
Changing the payload position in the previous manner did not affect the cart linear displacement much as noted in Figures 10(b) and 11(b). This is not expected to still happen for longer pendulum rod. However, the effect was more due to the value of the disturbance force applied on the rod.

The control effort seems to have more fluctuations when the payload is closer to the origin as noted in Figures $10(\mathrm{c})$ and $11(\mathrm{c})$.

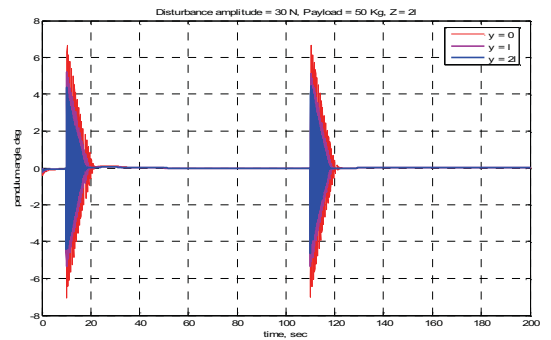

(a) Intermediate body tilt angle

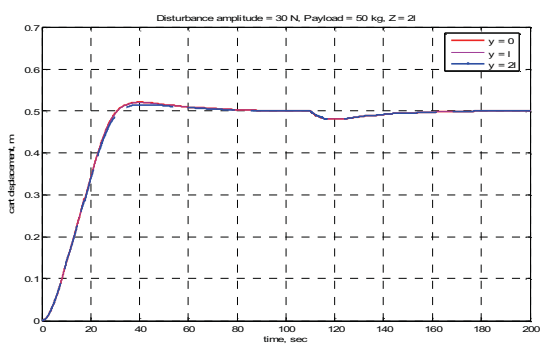

(b) Linear displacement of the vehicle wheels

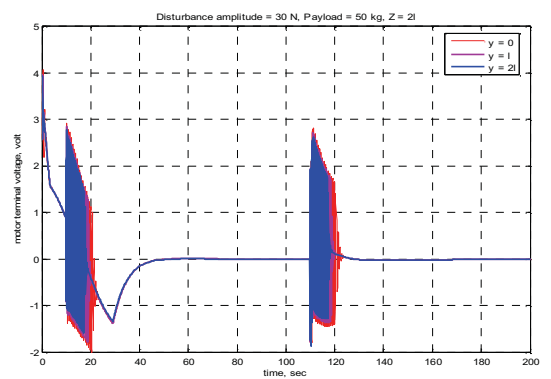

(c) Controller output signal to the system

Figure 11: System performance with load positions and $30 \mathrm{~N}$ disturbance force

\section{CONCLUSIONS}

The mathematical model of a robotic machine system with two wheels has been developed. An external disturbance force has been considered for application to the IB at different locations, and a payload has been attached to the rod. PD controller has been used to achieve the system performance target. The PD controller has been implemented on the developed model of the two-wheeled robotic machine in the balancing mode. Simulations have been carried out for different cases of changing three variables namely; the disturbance force level, the time duration of the 
disturbance force and the position of the payload. The system dynamic behaviour has been presented with control effort for achieving the system performance target in those different cases. It has been noted that the developed control algorithm can cope well in stabilising and balancing the system under different scenarios involving variations in the disturbance force value and duty cycle, and load position.

\section{REFERENCES}

D'Andrea R., and M.G. Earl. 2005. 'Design and Implementation of a Minimum Time Transition for an Inverted Pendulum". In Proceedings of the Asian Conference on Industrial Automation and Robotics, Bangkok, Thailand.

Formal'Skii A.M., and Y.G. Martynenko. 2005. "The Theory of the Control of a Monocycle". Journal of Applied Mathematics and Mechanics, 69 (4), 516528.

Goher K.M.K. and M.O. Tokhi. 2008. "Balancing of a Two-Wheeled Robotic Machine with Payload using PD/FL Controllers". In Proceedings of the $27^{\text {th }}$ IASTED international Conference, (Innsbruck, Austria, Feb. 11-13), 226-231.

Kim Y., H.K. Kim, and Y. Kwak. 2006. "Improving driving Ability for a Two-Wheeled Inverted Pendulum-Type Autonomous Vehicle". In Proceedings of IMechE.

Kim Y., H. K. Kim, and Y. K. Kwak. 2005. "Dynamic Analysis of a Nonholonomic Two-Wheeled Inverted Pendulum Robot". Journal of Inteligent and Robotic Systems, 44(1), 25-44.

Randal H.K.C. 2005. "Balancing Wheeled Robot". Research Project Toward the Degree of the Bachelor of Engineering, Faculty of Engineering and Surveying, University of Southern Queensland,

Salerno A., J. Angles. 2004. "The Control of SemiAutonomous Self Balancing Two-Wheeled Quasiholonomic Mobile Robot". Romanc.

Snike W.E., M.G.J. Gazdenman, and A. L. Hof. 2005. "The Condition of Dynamic Stability". Journal of BioMechanics, 38(1), 1-8.

Stan S., V. Maties, and R. Balan. 2005. "A Solution of the Inverted Pendulum on a Cart Problem Using Predictive Control". In Proceedings of the IEEE ISIE, Dubrovnik, Croatia.

Tsai C.C., S.C. Lin, and W.L.K. Luo. 2006a. "Adaptive Steering of a Self-Balancing Two-Wheeled Transporter". In Proceedings of 2006 Automatic Control Conference, Tamsui, Taiwan.

Tsai M.C. and J.S. Hu. 2006b. "Robust Control of AutoBalancing Two-Wheeled Cart Motion Pilot". In Proceedings of 2006 Automatic Control Conference, Tamsui, Taiwan.

\section{AUTHOR BIOGRAPHIES}

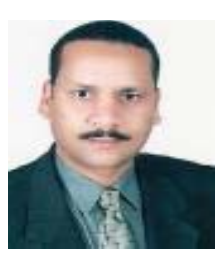

KHALED M K GOHER was born in Egypt. He studied the Mechanical Engineering at ElMinia University where he got his BSc. Degree in 1997. In 2005, he got his MSc. Degree in the same Institution in Dynamic Analysis of Stewart Parallel Manipulators. He worked as a demonstrator and assistant lecturer at the Department of Production Engineering and Mechanical Design, Faculty of Engineering, Suez Canal University, Egypt from 2000 to August 2006. Currently, he is doing his research toward the $\mathrm{PhD}$ at the Automatic Control and Systems Engineering Department, the University of Sheffield, UK. His main research interests are in mobile robotics, dynamic systems, intelligent control techniques, assistive technologies and mechatronics applications. Email address: k.mourad@shef.ac.uk

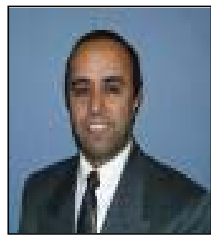

OSMAN TOKHI obtained his BSc (Electrical Engineering) from Kabul University (Afghanistan) in 1978 and $\mathrm{PhD}$ from Heriot-Watt University (UK) in 1988. He has worked as lecturer and Senior Lecturer in Kabul University, Glasgow College of Technology (UK) and the University of Sheffield (UK) and as sound engineer in industry. He is currently a Reader in the Department of Automatic Control and Systems Engineering, The University of Sheffield (UK). His main research interests include active control of noise and vibration, adaptive/intelligent control, soft computing techniques for modelling and control of dynamic systems, high-performance computing for realtime signal processing and control, and biomedical applications of robotics and control. He has extensive publications including textbooks, journal and conference papers in these areas. Email address: o.tokhi@shef.ac.uk http://www.sheffield.ac.uk/acse/staff/mot 